Boletín de la Sociedad Geológica Mexicana

Volumen Conmemorativo del Centenario

Aspectos Históricos de la Geología en México

Tомо LVII, NÚM. 2, 2005, P. 137-147

\title{
La Paleontología mexicana; pasado, presente y futuro
}

\author{
Ana Luisa Carreño*, Marisol Montellano-Ballesteros \\ Departamento de Paleontología, Instituto de Geología, Circuito Exterior, Ciudad Universitaria, \\ Delegación de Coyoacán 04510 D.F., México. \\ *anacar@servidor.unam.mx
}

\section{Prólogo}

Se presenta un panorama sobre las primeras menciones a propósito de los vestigios de vida en el pasado encontrados en el suelo y subsuelo de lo que hoy día constituye la República Mexicana, así como la historia — contada a grandes pasos- del desarrollo de la Paleontología en México.

No ha sido posible relatar los numerosos eventos que contribuyeron en la evolución de esta disciplina en el país debido, en gran medida, al objetivo del presente trabajo, que dista mucho de constituir una revisión histórica detallada de la paleontología mexicana y que se centra en delinear nuestros orígenes y nuestro de venir.

La tarea más ardua a la que nos enfrentamos durante la redacción de este documento fue decidir, entre los trabajos paleontológicos publicados e inéditos y resúmenes de los muchos autores prominentes y prolijos con los que esta disciplina ha contado y, afortunadamente cuenta aún, cuáles debíamos citar. Por ello, lanzamos un hilo conductor a lo largo del tiempo, y fue a través de él que fuimos ensartando algunos de los clásicos, los incunables, los que por muchos años, todavía, serán referencia obligada.

Finalmente, esperamos haber plasmado a grandes rasgos cómo la visión y el tesón de los grandes naturalistas, maestros, hombres y mujeres, forjaron individuos, que en un esfuerzo colectivo, todos los que conforman la historia de la paleontología en México, se han constituido en los pilares de la paleontología del Nuevo Milenio.

\section{El principio}

De acuerdo con revisiones recientes, los fósiles son conocidos desde la época prehispánica donde tuvieron una connotación mitológica; fueron empleados en las ofrendas de los rituales religiosos que se realizaban en algunos sitios mayas, o simplemente fueron empleados en la construcción de artefactos diferentes (Montellano-Ballesteros, 1999).

Después de la Conquista, la exploración del Nuevo Mundo también arrojó al encuentro no sólo de nuevas y diferentes culturas, sino también al hallazgo de restos de animales y plantas desconocidos para los conquistadores, dejando cuenta de éstos Antonio Herrera y Tordesillas (1615), quien menciona huesos muy grandes (elefantes) recolectados en México. De igual forma, el jesuita José Torrubia (1754) hace referencia a fósiles y minerales recolectados por él en el Nuevo Mundo.
Sin duda, una de las primeras menciones escritas sobre la presencia de fósiles en el territorio mexicano, que incluye una interpretación de los fenómenos geológicos asociados con su presencia, se encuentra en los escritos del misionero jesuita Miguel del Barco (1757), donde da cuenta de la presencia de conchas marinas en lugares alejados de las costas e incluso a grandes altitudes, relacionando esta presencia con la posibilidad de una regresión marina o, más aún, con la elevación del continente que dejó al descubierto el lecho marino.

Durante la Colonia y hasta el siglo XVIII, estos restos de vida pasada despertaron cierto interés, existiendo un número importante de reportes inéditos, como resultado de las exploraciones mineras realizadas en el país por compañías extranjeras, en los que se menciona la presencia de fósiles pero sin hacer un estudio detallado de ellos y sin asignar un valor a su presencia por no estar claramente asociados con 
los yacimientos objeto de la exploración. A pesar de ello, algunos hallazgos fósiles fueron documentados por revistas que se encargaban de popularizar éstos como en el caso de la presencia de vertebrados fósiles de México (Anónimo, 1799), publicación que, por otro lado, constituye la mención documentada más antigua para este grupo.

Con la constitución en 1792 del Real Seminario de Minería en la Ciudad de México, varios científicos extranjeros fueron invitados a participar en las actividades de enseñanza. Entre ellos destaca Andrés Manuel del Río (1795) por su obra Elementos de Orictognosia.

A pesar de las referencias antes mencionadas, es hasta el siglo X IX cuando los trabajos hechos por especialistas, fundamentalmente extranjeros, dan a los fósiles y su estudio un carácter netamente científico (Gío-Argáez, 2004). Sin embargo, aún es frecuente encontrar citas a fósiles y minerales como parte de exploraciones del territorio nacional organizadas con fines variados, como es el reporte de Juan de Obregoso, publicado en 1931.

Los primeros estudios con carácter eminentemente científico publicados corresponden, en su mayoría, a autores extranjeros, entre los cuales cabe mencionar los de los belgas Galeotti (1839) y Nyest y Galeotti (1840) sobre la presencia de invertebrados de la región de Tehuacán, Puebla y en los alrededores de Jalapa, Veracruz. A partir de esta fecha, son frecuentes las referencias a estudios sobre material fósil proveniente de diversas localidades del país como el de Von Meyer (1840a, b) sobre vertebrados del Valle de México; los de Gabb (1864, 1869) en Sonora; Gabb (1872) en Chihuahua; así como Cotteau (1890), por mencionar algunos autores.

La importante pérdida de territorio como resultado de la invasión estadounidense a México a mediados del siglo XIX (1846-1847), obligó al gobierno de ese país a formar, bajo el mando del Mayor W. H. Emory, una comisión encargada de trazar la nueva frontera entre ambos países. Esta comisión, integrada por J.R. Bartlett, J. Hall, A. Schott y T. Conrad, publica un informe incluyendo la estratigrafía, petrografía y, por supuesto, la paleontología (Conrad, 1857).

Una importante contribución sobre los fósiles de México, resultado esta vez de la intervención francesa a México (1862-1867), fueron los tres volúmenes de los Archives de la Commission Scientifique du Mexique (1867), elaborados por la Mission Scientifique au Mexique et dans l'Amérique Centrale, en la que participaron el zoólogo M. Mine Edwards, así como los geólogos G. Tarayre, A. Dollfus, E. de Montserrat y P. Pavie.

Hacia finales del siglo XIX, un número importante de biólogos, naturalistas y geólogos, particularmente extranjeros, demuestra gran interés por la geología y la paleontología de México publicando sus hallazgos en revistas extranjeras a la manera de pequeños informes. Trabajos científicos relevantes de finales de ese siglo son los de Cope (1872, 1884, 1886a, b,1893), quien describe especímenes importantes de vertebrados resguardados en la colección

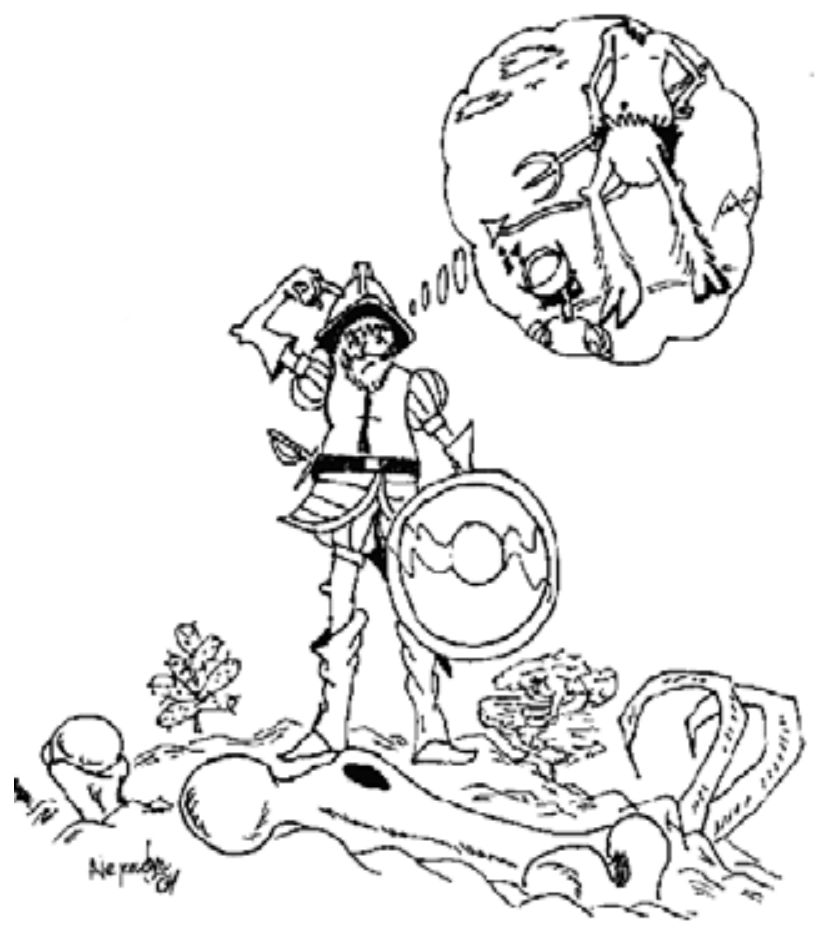

de la Escuela Nacional de Ingenieros. También por ser los primeros trabajos donde participan naturalistas mexicanos y/o que fueron escritos en español, vale la pena mencionar los de Cuatáparo y Ramírez (1875), quienes apuntan la presencia de un "Glyptodon" en el Distrito de Zumpango y los de Dugès $(1882,1891)$ quien registra la presencia de vertebrados fósiles en el estado de Guanajuato y describe a Platygonus alemanii y restos de organismos de origen sudamericano.

Aunque mucho más escasos, también encontramos mención a la presencia en territorio nacional de plantas fósiles, como es el caso de los trabajos de Martins (1871) y Grisebach (1883).

Estas últimas publicaciones son relevantes, no sólo por la temática de las mismas, sino porque fueron publicadas en la revista La Naturaleza, órgano oficial de difusión científica la Sociedad Mexicana de Historia Natural (1868), que junto con la Sociedad Científica Antonio Alzate, que más tarde sería la Academia Nacional de Ciencias en México, constituyen las sociedades de finales del siglo XIX y principios del XX que contribuyeron de manera importante al pensamiento científico, de las aún incipientes disciplinas de las ciencias naturales que se desarrollaban en México, como la Paleontología.

Por supuesto, mención especial requiere por su aporte científico el trabajo realizado por varios autores extranjeros a finales del siglo XIX y que fuera publicado en las obras compiladas por Felix y Lenk (1889-1899), entre las que cabe mencionar, por la escasez de este tipo de investigación en la época, la de Nathorst (1899) sobre la presencia de plantas fósiles de Tlaxiaco. 


\section{La creciente institucionalización y el ímpetu petrolero}

Los esfuerzos pioneros encaminados a formar las primeras colecciones científicas en paleontología, dan un impulso importante a esta disciplina a finales del siglo XIX, despertando, por otro lado, gran interés por la misma. Una de las iniciativas más importantes dentro de este contexto ocurre en 1886, fecha en que por decreto del Congreso de la Unión, se crea la Comisión Geológica y, dos años después, se aprueba la fundación del Instituto Geológico, designando al Ing. Antonio del Castillo como su director, quien dispone la organización y arreglo de lo que serían las primeras colecciones paleontológicas permanentes de nuestro país. Estas colecciones incluyeron los registros de vertebrados fósiles publicados por Richard Owen (1869), así como los de los primeros invertebrados descritos por el eminente geólogo jalisciense Mariano Bárcena (1875a, b) y los fósiles de mamíferos listados por el propio del Castillo $(1869,1879)$. En esta colección, también fueron alojados materiales extranjeros, principalmente de Europa, así como material que fue recolectado y adquirido por los investigadores del Instituto.

A la muerte de del Castillo en 1895, José Guadalupe Aguilera-Serrano publica el primer número del Boletín del Instituto Geológico "Fauna fósil de la Sierra del Catorce", el cual se convierte en la publicación que daría a conocer por más de una centuria, los estudios que realizaba el personal del propio Instituto. Aguilera-Serrano no sólo contribuyó de esta forma con el desarrollo de la Paleontología, sino que también impartió cursos de esta disciplina en la Facultad de Altos Estudios de enero de 1923 hasta diciembre de 1924 y en la Escuela de Ingenieros de 1934-1949.

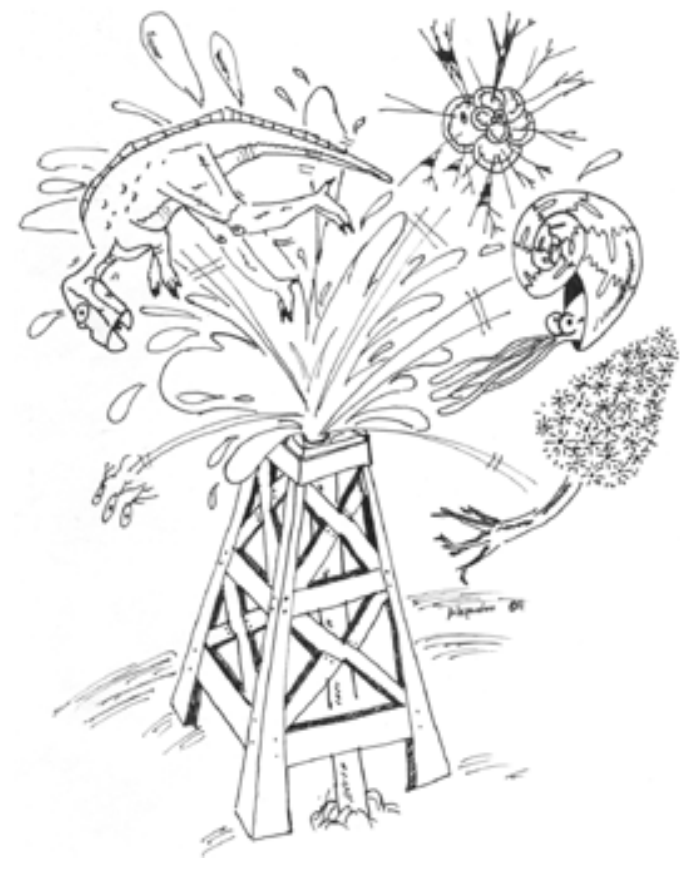

Aun cuando la existencia de aceite mineral en la República Mexicana se conocía desde la época prehispánica e incluso había referencias escritas en relación con el petróleo por Fray Bernardino Ribeira (Fray Bernardino de Sahagún) en su Historia General de las Cosas de la Nueva España (1558-1569), los primeros intentos de aprovechamiento industrial datan de 1863, año en que el sacerdote Manuel Gil y Sáenz descubre una exudación de petróleo cerca de Tepetitlán, Tabasco y pretende su explotación sin éxito.

Es en 1900 que inicia la búsqueda por hidrocarburos, cuando Charles A. Candfield y Edward L. Doheny compran la hacienda El Tulillo (estados de San Luis Potosí, Tamaulipas y Veracruz), que más tarde sería propiedad de la Mexican Petroleum of California. Esta empresa, creada por el propio Doheny, perfora en el campo El Ébano, extrayendo petróleo en 1901, mediante un pozo bautizado como Doheny I. Más tarde (1904), en la zona de Cougas (Furbero), con la perforación del pozo La Pez núm. 1 de la Mexican Petroleum Company se inicia, en gran escala, la exploración y explotación por hidrocarburos. Con esta industria, se instala en nuestro país a principios del siglo $\mathrm{XX}$ un número importante de compañías petroleras extranjeras (Compañía Mexicana de Petróleo El Águila, S. A., Compañía Naviera de San Cristóbal, S. A., Compañía Naviera San Ricardo, S. A., Huasteca Petroleum Company, Sinclair Pierce Oil Company, Mexican Sinclair Petroleum Corporation, Stanford y Compañía, S. en C. Peen Mex Fuel Company, Richmond Petroleum Company de México, California Standard Oil Company of Mexico, Compañía Mexicana el Agwi, S. A., Compañía de Gas y Combustible Imperio, Consolidated Oil Company of México, Compañía Mexicana de Vapores San Antonio, S. A., Sábalo Transportation Company, Clarita, S. A. y Cacalilao, S. A., entre otras), y con ellas se inicia también la aplicación de la paleontología a la búsqueda por hidrocarburos.

Es durante este período que micropaleontólogos de instituciones extranjeras como Cushman (1920, 1925, 1926, 1927), Cole (1927, 1928a, b), Cole y Guillespie (1930), Nuttall $(1928,1930,1932)$, White (1928a, b), Galloway y Morrey (1931), Barker (1939) y Vaughan y Cole (1936), entre otros, fungen como consultores de algunas de las compañías petroleras que operaban en nuestro país.

El trabajo desarrollado por estos consultores fue intenso y es por ello que en este período muchas de las revistas extranjeras dedicadas a dar a conocer los hallazgos paleontológicos relevantes, como el Journal of Paleontology y el Bulletin of American Paleontology, por mencionar algunas, incluyen numerosos artículos que dan cuenta de nuevas especies de fósiles encontrados en suelo y subsuelo mexicano. De llamar la atención es el primer número de la importante revista dedicada exclusivamente a los foraminíferos y fundada por Alfred Cushman, Contributions of Cushman Laboratory for Foraminiferal Research, que en su primer número hace la mención de los foraminíferos del Eoceno de México. 


\section{El siglo XX y los primeros paleontólogos}

Hasta mediados del siglo XX y con el ímpetu petrolero y minero, México se convierte en el receptor de numerosos paleontólogos de nacionalidades varias. Algunos llegan para trabajar por solicitud del gobierno federal, otros como parte de expediciones científicas de sus respectivos gobiernos. La extensa bibliografía que sobre fósiles mexicanos se encuentra publicada da cuenta de lo prolífico de la obra de estos personajes, entre los que no se puede dejar de nombrar, por el carácter monográfico y descriptivo de un número importante de nuevas especies de invertebrados para México, las de Böse (1910, 1918, 1923, 1927, entre otras), Burckhardt (1906, 1912, 1919, 1925, 1927), Hertlein $(1925,1934)$ e Imlay (1937a, b, 1938, 1939, 1940, por mencionar algunas). Durante este mismo intervalo de tiempo, los trabajos sobre fusulínidos por Dunbar $(1939,1944)$ y de vertebrados continentales de Freudenberg (1910, 1921, 1922) ocupan un lugar importante dentro de la paleontología clásica para México, así como los trabajos de Berry (1923), Noé (1937) y Weyland (1937), que son de los pocos que incluyen información sobre plantas fósiles.

Muchos otros paleontólogos permanecieron hasta el fin de sus días trabajando en México, como en el caso del alemán Federico Müllerried (1931,1933, 1944, 1945, 1947, 1951, entre muchos), quien llega en 1922 y trabaja en instituciones nacionales y de América Central, entre las que destacan el Instituto Geológico, el Instituto de Biología y el Museo Nacional de Historia Natural donde estuvo a cargo de las colecciones paleontológicas y biológicas.

\section{El gran impulso}

Varios acontecimientos parecen haber impulsado la formación de paleontólogos mexicanos. Uno de ellos es la iniciativa de la comunidad paleontológica, con sede en el Instituto de Geología de la UNAM y de su director el Ing. M. Santillán que, con la mediación del Rector Ignacio García Téllez, solicitan al Presidente de la República, C. Pascual Ortiz Rubio, que los duplicados de fósiles y minerales, que estaban resguardados por la Secretaría de Agricultura y Fomento con base en un acuerdo firmado en 1927, fueran enviados a la Universidad Nacional Autónoma de México. De esta forma se empezó a consolidar la Colección Nacional de Paleontología.

Otro aspecto que impulsa la Paleontología en México probablemente fue la expropiación petrolera en 1938, y la consiguiente creación de Petróleos Mexicanos (PEMEX) para administrar y operar la industria petrolera nacionalizada. En el mismo sentido, el compromiso adquirido por el Instituto Politécnico Nacional y la Universidad Nacional Autónoma de México de formar los cuadros necesarios para atender las necesidades de la nueva industria petrolera mexicana, favoreció el desarrollo de esta disciplina.
De esta forma, por iniciativa de E. Díaz Lozano en PEMEX, fue creada una sección de Paleontología, aunque buena parte del trabajo paleontológico realizado durante los primeros años quedó inédita.

La creación de los Anales de la Escuela Nacional de Ciencias Biológicas y del Instituto de Biología (Maldonado-Koerdell, 1948, 1950, 1953a, b); la aparición de la serie Paleontología Mexicana (Erben, 1954; Alencaster, 1956) como una publicación formal del Instituto de Geología; la organización de la Sección de Paleontología durante los trabajos del XX Congreso Geológico Internacional (Erben, 1956), así como la inclusión de artículos paleontológicos en los boletines de la Asociación Mexicana de Geólogos Petroleros (Alencaster, 1950; Bonet, 1956) y de la Sociedad Geológica Mexicana, contribuyeron a la difusión de los hallazgos de los paleontólogos extranjeros todavía en nuestro país y de los biólogos y geólogos que exploraban esta nueva disciplina.

Hacia mediados y durante principios de la segunda parte del siglo pasado, la creación en 1965 del Instituto Mexicano del Petróleo (IMP) y de su revista vino, por un lado, a consolidar la tradición en Micropaleontología (Trejo, 1969, 1972) y, por el otro, abrió nuevamente espacio para la paleontología de invertebrados como arma en la industria petrolera (Cantú-Chapa, 1967, 1968).

Pronto, las revistas que habían visto la luz a principios del siglo comienzan a publicar más trabajos de invertebrados (Buitrón, 1970, 1971, 1973; Ochoterena, 1960, 1963, 1966; Perrilliat, 1972, 1973, 1974a, b, 1976, 1977, 1984; Reyeros de Castillo, 1974, 1978), de vertebrados (CarranzaCastañeda, 1978; Ferrusquía-Villafranca, 1969, 1978; Mooser, 1958, 1959, 1963) y de plantas fósiles (Weber, 1972, 1973,1975; Silva-Pineda, 1970, 1978), por citar sólo las obras clásicas de estos autores.

Del lado de los petroleros, también se puede citar aportaciones importantes como las de Obregón de la Parra (1958, 1959), Salmerón (1972) y Sansores y FloresCovarrubias (1972).

Para esta época, ya se había plantado varias semillas en las instituciones de educación superior del país. La cátedra de Paleontología en el Instituto Politécnico Nacional y en la Universidad Nacional Autónoma de México, empezaba a dar sus primeros frutos $\mathrm{y}$, con ello, numerosas tesis de licenciatura en ambas instituciones empiezan a generar lo que constituiría la planta académica de instituciones como el Instituto Mexicano del Petróleo, el Instituto de Geología de la UNAM y el Instituto Nacional de Antropología e Historia.

Debido a que desde sus inicios los arqueólogos estudiaban la presencia temprana del hombre en México, el Instituto Nacional de Antropología e Historia, por medio del entonces Departamento de Prehistoria y desde 1963 con la creación del Laboratorio de Paleozoología, tiene una larga historia en el desarrollo de una importante colección paleontológica constituida básicamente por restos provenientes del último periodo geológico y de un plantel 
de profesionales dedicados al estudio de la arqueoflora y arqueofauna mexicana (Polaco y Arroyo-Cabrales, 1991).

En PEMEX y el IMP se instala el cuerpo académico más completo en cuanto a Micropaleontología se refiere y, aunque la mayoría de sus contribuciones permanece como trabajos inéditos, su aportación al campo de la paleontología aplicada es innegable.

\section{Un giro importante}

A finales de la década de los 70 ocurre un cambio importante en el estudio de la paleontología. Hasta esta época, y por razones por demás obvias, la mayoría de los trabajos publicados era descriptiva o bien tenía una clara implicación bioestratigráfica.

El apoyo brindado para realizar posgrados en el extranjero trajo como consecuencia nuevas visiones sobre qué y cómo hacer en paleontología. También hubo una creciente colaboración con especialistas extranjeros, lo que favoreció el trabajo multi- e interdisciplinario, creándose nuevas líneas de investigación. De esta forma, la Palinología empieza a tomar su lugar en la historia (Salas y Palacios-Fest, 1982; Martínez-Hernández et al., 1980) así como el estudio de vertebrados marinos (Applegate, 1978; Espinosa-Arrubarrena y Applegate, 1996), entre otras disciplinas.

La formación de nuevos centros de investigación, como el de Ciencias del Mar y Limnología, favoreció la diversificación de algunas áreas de la micropaleontología como la oceanográfica, incentivada por los Drs. A. AyalaCastañares y R. Segura-Vernis.

Algunos eventos marcan de manera decisiva a los paleontólogos mexicanos: el primero de ellos, en 1982, fue la realización fuera de los Estados Unidos de América, del 42 congreso anual de la Society of Vertebrate Paleontology. Fue la primera ocasión en que se acogió a colegas paleontólogos para discutir e intercambiar ideas dentro de una reunión exclusiva para el gremio. Otro evento importante fue la Reunión del Programa Internacional de Correlación Geológica (IGCP) de los proyectos 157 y 160, donde se reunieron estudiosos del Precámbrico. Asimismo, el III Congreso Latinoamericano de Paleontología, llevado a cabo en 1983 en Oaxtepec, Morelos, reunió a lo más prestigiado de las diferentes disciplinas en América Central y, particularmente, de América del Sur.

La paleontología ya no era parte de otra reunión o congreso, era el evento y, así, la paleontología mexicana ocupaba su lugar en el orden mundial.

Es en 1986 que se constituye formalmente la Sociedad Mexicana de Paleontología, agrupando alrededor de 100 paleontólogos mexicanos, algunos extranjeros y aficionados. Esta sociedad permitió con su revista y congreso bianual que hubiera una difusión de las investigaciones en curso por los diferentes grupos de trabajo, y también la colaboración estrecha entre instituciones. Años más tarde, el congreso abandonaría la Ciudad de México, y sería acogido por los paleontólogos de Linares (UANL), Guadalajara (INAH) y Chiapas (INHE), mientras esperan su turno Ensenada (UABC) y otras instituciones de investigación y enseñanza superior del país.

\section{El Consejo Nacional de Paleontología}

Históricamente y a pesar de la amplia tradición en paleontología en cuanto al desarrollo de colecciones científicas, las leyes y reglamentos relacionados con el patrimonio paleontológico no habían tenido el fundamento ni la consistencia jurídica que sustentara su protección, debido a que, de 1927 a 1965, este patrimonio había estado, aunque en ocasiones sólo implícitamente, bajo la custodia de algunas instituciones gubernamentales que, a lo largo de la historia reciente del país, se han encargado de diversos aspectos de la cultura en México (Secretaría de Industria y Fomento, Agricultura y Fomento, Secretaría de Educación Pública).

Los antecedentes más concretos se sitúan en 1970, cuando se publica la Ley Federal del Patrimonio Cultural de la Nación, dentro de la cual se incluía el material paleontológico, tal vez por vez primera, de manera explícita y formal.

Es hasta 1986 que se publica un decreto presidencial donde se reforma la Ley Orgánica del Instituto Nacional de Antropología e Historia (INAH), con la adición del Artículo 28 bis. De esta forma, el patrimonio paleontológico queda bajo la jurisdicción absoluta de la Secretaría de Educación Pública (SEP) a través del propio INAH.

Para tratar de entender, normar criterios sobre estos aspectos y poder legislar salvaguardando, por otro lado, las leyes mexicanas que conceden el régimen de propiedad privada para la superficie de un terreno pero no para el subsuelo, en 1994 el INAH constituye el Consejo Nacional de Paleontología, con la idea de que un grupo multidisciplinario e interinstitucional pudiera llegar a un acuerdo sobre qué, cómo y porqué legislar todos estos aspectos. Este Consejo se constituyó con la invitación que hiciera el director del Instituto Nacional de Antropología e Historia a las instituciones de educación superior e investigación.

El Consejo quedó integrado por la Universidad Nacional Autónoma de México -que delegó la responsabilidad en el Director del Instituto de Geología, por ser ésta la dependencia universitaria que tenía bajo su protección la Colección Nacional de Paleontología- la Universidad Autónoma de Baja California, el Instituto Politécnico Nacional, la Universidad Autónoma de Nuevo León, el Instituto Mexicano del Petróleo y la Sociedad Mexicana de Paleontología. Este Consejo sesionó por varios meses y elaboró un documento que fue la base de una iniciativa de decreto en el ámbito federal, para legislar sobre el patrimonio paleontológico. 
Por supuesto, hubo que definir los diferentes tipos de fósiles, tomar en consideración las actividades económicas rentables para el país y, finalmente pero no menos importante, que la Paleontología es definitivamente Patrimonio Universal.

El documento fue presentado ante la Comisión de Cultura de la LVII Legislatura del Senado de la República, que formuló la iniciativa propiamente dicha. Ésta, a su vez, fue turnada a la Cámara de Diputados y actualmente se encuentra en revisión por las Legislaturas Locales de cada estado de la República.

\section{La academia}

La creación de nuevas entidades académicas de educación superior que incluían la enseñanza de la paleontología en su curricula (i.e., Universidad Autónoma Metropolitana, CICESE, Universidad Autónoma de Baja California, Universidad Autónoma de Baja California Sur, Universidad Autónoma de Nuevo León campi Linares y Monterrey, Universidad Autónoma de Sonora, Universidad Autónoma del Estado de Morelos y Universidad Autónoma de Guerrero) favoreció el incremento en líneas de investigación y abrió nuevas posibilidades (Universidad Autónoma del Estado de Hidalgo, Universidad Autónoma del Estado de Chiapas, Universidad del Mar en Oaxaca y la Universidad Michoacana de San Nicolás de Hidalgo, por mencionar algunas).

De acuerdo con las cifras proporcionadas por GíoArgáez (2004), durante la segunda parte del siglo XX se produjeron aproximadamente 465 publicaciones de autores extranjeros y alrededor de 941 de autores nacionales, con un total desde el año de 1839, de 1720 referencias.

Un cambio sustancial se registra a mediados de la década de los noventa, cuando gran parte de las contribuciones paleontológicas se encuentra en publicaciones extranjeras, y cada vez más frecuentemente, tratando aspectos como la anatomía vegetal (http://geologia.igeolcu.unam.mx/PALEO/cevallos/index.html), cambio climático (http://geologia.igeolcu.unam.mx/paleo/slozano.htm; http://igeofcu. unam.mx/geomag/paleoamb/maga.html; http://smm.iim. umich.mx/depgeologia/isa\%5Cproyectos2003.htm), evolución del Golfo de California (http://geologia.igeolcu. unam.mx/PALEO/ACarreno/carhoja.htm), tafonomía de invertebrados (http://oceanologia.ens.uabc.mx/facultad/ personal/tellez/htm) y Ecoestratigrafía (http://geologia. igeolcu.unam.mx/paleo/anaberta.htm).

También algunos grupos que eran prácticamente desconocidos en México, salvo por algunos trabajos aislados hechos por extranjeros, empiezan a conocerse. Entre ellos se puede citar los de radiolarios ( $†$ A. Molina-Cruz), de dinosaurios (Hernández-Rivera, 1997), de dinoflagelados (http://geologia.cicese.mx/jhelenes/helenes.html), de crustáceos (http://geologia.igeolcu.unam.mx/geol/fvega. htm), de vertebrados marinos (http://oceanologia.ens.uabc. $\mathrm{mx} /$ facultad/personal/aranda/htm), de peces (http:// www. reduaeh.mx/investigación/biología/investigadores/ganzales_Rodríguez.htm), de reptiles (http://www.ibiologia. unam.mx/directorio/ReynosoRosales.htm) y sobre la evolución morfológica floral de angiospermas (http://www. ibiologia.unam.mx/directorio/m/magallon_puebla.htm).

Se desarrollan otras líneas de investigación y comienza la utilización de algunos microfósiles como indicadores de contaminación ambiental o de cambios bruscos en las condiciones ambientales (M.R. Palacios-Fest: Terra Nostra - Earth Science Research), así como el empleo de técnicas geoquímicas para la interpretación de aspectos paleobiológicos, y la aplicación del paleomagnetismo en la cronología de vertebrados (M. Benammi).

Petróleos Mexicanos y el Instituto Mexicano de Petróleo continúan desarrollando su labor con microfósiles calcáreos y bioestratigrafía de alta resolución, brindando con su labor cotidiana un apoyo importante a la exploración por hidrocarburos en el país, en momentos cuando el boom petrolero es parte de la historia.

La docencia se ve muy beneficiada, particularmente en facultades y centros dependientes de la UNAM, del Instituto Politécnico Nacional, centros de educación superior SEP-CONACyT y del Instituto Nacional de Antropología e Historia. Un ejemplo de ello es la creación del Museo de Paleontología de la Facultad de Ciencias de la UNAM ( http://www.fciencias.unam.mx/Museo_De_ Paleontologia).

\section{El límite y-K2}

Hacia finales del siglo XX, México se convierte en el centro de un antiguo debate. La comunidad internacional de ciencias de la Tierra es informada de que en un lugar llamado Chicxulub, en el estado de Yucatán, México, hay pistas que podrían llevar al encuentro del asesino sideral de la biota de finales del Cretácico. La búsqueda de evidencias del gran tsunami, los cuarzos de impacto, el cálculo de las medidas del posible infractor, los retratos hablados, la teoría del asesino solitario vs. varios cómplices, todo ello, revive el gran debate ancestral sobre el paradigma de las extinciones (Carreño y Montellano-Ballesteros, 1997). Biólogos, paleontólogos, geólogos, geofísicos y astrónomos debaten como nunca sobre el asunto. El tema proporciona una intensa exploración y una producción prolija sobre todos los tópicos. No existe congreso o reunión importante en el mundo que no incluya un simposio para discutir el gran descubrimiento. Todos toman partido. No hay duda, la huella del asesino está ahí. Infortunadamente, quedan más preguntas que respuestas.

Nuevos hallazgos en todos los ámbitos de la paleontología ocurren día a día, otros se revisan a la luz de las nuevas técnicas y conocimientos. Los paleontólogos en ciernes egresados de las licenciaturas en Biología y Geología llegan a los centros de educación superior e investigación motiva- 
dos por sus clases, pero también, en gran medida, atraídos por la amplia difusión que de los fósiles han realizado los medios masivos de comunicación. Un ejemplo de ello, son las películas Parque Jurásico e Impacto Profundo y, más recientemente, La Edad de Hielo y El Día Después de Mañana, donde la paleontología, sus teorías y sus debates son puestos al alcance de la sociedad.

Aunque cada día el país cuenta con más paleontólogos, aún son insuficientes para cubrir las necesidades académicas. Por ello, muchos de los grupos de investigación cuentan con el apoyo de distinguidos paleontólogos extranjeros como F. Oloriz de la Universidad de Granada; J. M. Pons de la Universidad Autónoma de Barcelona; Consuelo Díaz Otero y José Fernández Carmona del Instituto de Geología y Paleontología de la Habana y del Centro de Investigaciones del Petróleo en Cuba, respectivamente; D. Vachard de la Universidad de Lille; D. Fastovsky de la Universidad de Rhode Island; J. Lipps de la Universidad de California, Berkeley; W. Miller de la Universidad Bringham Young; João C. Coimbra de la Universidad Federal De Rio Grande do Sul, por mencionar algunos.

Menos espectacular, pero igualmente motivante, es el impulso que han recibido museos como el de Geología (http://geologia.igeolcu.unam.mx/MuseoPrueba2.htm\#) y la creación de otros denominados de sitio como el de Tepexi de Rodríguez (http://www.geocities.com/ tepexiderodriguez), en el estado de Puebla.

Por supuesto que los gobiernos estatales han puesto su grano de arena, y en la última década han apoyado la construcción de museos de historia natural donde no podía faltar la sección de Paleontología. En éstos, se muestra la riqueza fosilífera del estado en cuestión y se da un vistazo a la historia de la vida.

Buenos ejemplos de este esfuerzo son el Museo del Desierto, Coahuila (http://www-museodeldesierto.org/inicio.htm), Museo Regional de Historia de Aguascalientes (http://www.inah.gob.mx/muse2/htme/mure0101.html), Museo de Tamaulipas (TAMUX), Museo de Paleontología de Guadalajara Federico A. Solórzano Barreto (http://www. guadalajara.gob.mx/dependencias/museopaleontologia/index.html) y el Museo de Paleontología Eliseo Palacios en Tuxtla Gutiérrez, Chiapas (http://www.ihne.chiapas.gob. $\mathrm{mx} /$ investigacion/museo/asp) donde la labor de Don Miguel Álvarez del Toro, impulsor de los estudios de historia natural en el estado debe ser resaltada.

Estas acciones, junto con el papel que han jugado los aficionados - personas que tienen un sentimiento e interés especial por los fósiles-y que en algunos casos, con sus propios medios han financiado museos como el de $\mathrm{La}$ Laguna (http://www.torreon.gob.mx/laciudad/turismo/museos/paleontologia.php) y Sabinas (http://www.sabinas. gob.mx/turismo/browse_destino.asp?DESTINO_ID=27) en el estado de Coahuila, así como el de Delicias en Chihuahua (http://fosilesmexico.i8.com/principal.html), que es otro ejemplo de la gestión de aficionados ante los gobiernos estatal o municipal, han contribuido en su conjunto, por medio de exhibiciones, conferencias y actividades de difusión, a permear a la sociedad el conocimiento sobre la riqueza fosilífera en México y su importancia.

Ya sea por los medios masivos de información y comunicación, por el entusiasmo de académicos, docentes y neófitos, por las iniciativas estatales o incluso por el interés innato de ejidatarios, como la familia Aranguti de Tepexi de Rodríguez, Puebla, la cual reconociendo el valor de los fósiles encontrados en sus tierras decidió ponerlos en manos de los científicos para su resguardo, acción que le valió el reconocimiento internacional (Harroll L. Strimple Award, otorgado por The Paleontological Society, Morán, 1999), la paleontología en México sigue inexorablemente su curso natural.

Con la participación de muchos paleontólogos de los aquí nombrados, se ha formado la Colección Nacional de Paleontología, reconocida por instituciones nacionales y extranjeras, y actualmente miembro activo de la Natural Science Collections Alliance. Esta colección, alojada en el Museo de Geología M. Carmen Perrilliat, en Ciudad Universitaria, contiene 8,000 ejemplares en la Colección de Tipos; con 3, 400 localidades fosilíferas en la República Mexicana en la Colección Geográfica de Referencia y más de 8,000 especies (no ejemplares) en la Colección de Material Extranjero, así como un número importante de ejemplares en la Colección de Material Reciente.

\section{La perspectiva}

México, desde el punto de vista biológico, es un centro de origen, evolución, migración y extinción de muchos grupos a través del tiempo. Por ello, tiene una riqueza en flora y fauna fósil que motiva a propios y extraños a estudiar y comprender su historia.

Un número importante de los paleontólogos activos en investigación imparte actualmente cursos de licenciatura o de posgrado en las instituciones de educación superior del país y motiva con su cátedra a numerosos jóvenes a continuar descifrando la evolución de las biotas a través del estudio biológico de sus fósiles.

El interés por las nuevas metodologías y técnicas para realizar estudios taxonómicos, paleobiológicos y biogeográficos, entre muchos otros, está tomando cada día auge. La pasión por los dinosaurios despierta la imaginación de los estudiantes, mientras otros se fascinan por el mundo microscópico.

Otros muchos, comprenden que la paleontología es el jamón del sandwich y se interesan por la geología y sus modernas armas como la geoquímica, la estratigrafía por secuencia y la isotopía. Un camino extenso falta por recorrer antes de tener completa la historia y evolución geológica del país y en esa historia, no hay duda, la paleontología tiene un lugar preponderante.

En los albores del nuevo milenio, se aproxima un giro importante. Nuevas líneas de investigación están empezan- 
do a surgir, en parte debido a las necesidades de resolver problemas nacionales, o a que la tecnología permite ahora explorar otras áreas del conocimiento paleontológico. Una concentración importante de jóvenes paleontólogos se está formando en México, otros muchos, todavía en el extranjero.

Viejos paradigmas continúan vigentes. Muchas áreas de la paleontología permanecen prácticamente desconocidas. Otras, requieren de una revisión a la luz de las nuevas teorías.

A pesar de las nuevas tecnologías, muchas áreas permanecen, actualizadas sí, pero con el mismo valor de antaño. El peso de los fósiles como indicadores de edad no ha podido ser superado. De ellos depende una parte de nuestra economía y, por ello, debemos seguir promoviéndolos.

Junto con el Nuevo Milenio, llegan nuevos retos, y son los jóvenes entusiastas los que habrán de enfrentarlos. El compromiso de los paleontólogos que alcanzamos esta aurora va más allá de la transmisión de nuestros conocimientos; debemos formarlos para que la tradición continúe. Esa es la desiderata.

\section{Agradecimientos.}

Las autoras agradecen a M. Alcayde-Orraca, por la revisión de estilo del manuscrito. De igual forma agradecen al revisor quien con sus comentarios enriqueció sustancialmente el manuscrito.

\section{Referencias bibliográficas}

Alencaster, G., 1950, Nota sobre dentálidos del Terciario de México: Asociación Mexicana Geólogos Petroleros, Boletín, 2, 221-226.

Alencaster, G., 1956, Pelecípodos y gasterópodos del Cretácico Inferior de la región de San Juan Raya, Zapotitlán, Estado de Puebla: Universidad Nacional Autónoma México, Instituto Geología, Paleontología Mexicana, 2, 47 p.

Anónimo, 1799. Noticia sobre una osamenta de un elefante fósil: Gaceta de Méxicom, 9, 353.

Applegate, S.P., 1978, Phyletic studies, Part I: Tiger sharks: Universidad Nacional Autónoma México, Instituto Geología, Revista, 2(1), 55-64.

Bárcena, M., 1875a, Descripción de un crustáceo fósil del género Spheroma (S. burkartii) y reseña geológica del Valle de Ameca, Jalisco: La Naturaleza, 3, 355-361.

Bárcena, M., 1875b, Datos para el estudio de las rocas mesozoicas de México y sus fósiles característicos: Sociedad Mexicana Geografía Estadística, Boletín Ser. 3, 2, 369-405.

Barco, M. del, 1757, Historia Natural y Crónica de la Antigua California. Edición y estudio preliminar de M. León-Portilla, (1988): Universidad Nacional Autónoma de México, Instituto de Investigaciones Históricas, $482 \mathrm{p}$.

Barker, R.W., 1939, Species of the foraminiferal Famiy Camerinidae in the Tertiary and Cretaceous of Mexico: Proceedings U.S. Natural Museum, 86(3052), 305-330.

Barker, R.W., Grimsdale, T.F., 1936, A contribution to the phylogeny of the Orbitoidal Foraminifera, with description of new forms from the Eocene of Mexico: Journal of Paleontology, 10(4), 231-247.

Berry, E.W., 1923, Miocene plants from southern Mexico: Proceedings U.S. Natural Museum, 62(19), 1-27.
Bonet, F., 1956, Zonificación microfaunística de las calizas cretácicas del este de México: Asociación Mexicana Geólogos Petroleros, Boletín, 8(7-8), 389-488.

Böse, E., 1910, Monografía geológica y paleontológica del Cerro de Muleros cerca de Ciudad Juárez, Estado de Chihuahua y descripción de la fauna cretácica de la Encantada, Placer de Guadalupe, Estado de Chihuahua: Instituto Geología México, Boletín, 25, 193 p.

Böse, E., 1918 (1920), On a new ammonite fauna of the lower Turonian of Mexico: Texas University, Bulletin, 1856, 173-252.

Böse, E., 1923, Algunas faunas cretácicas de Zacatecas, Durango y Guerrero: Instituto Geológico de México, Boletín, 42, 219 p.

Böse, E., 1927, Cretaceous ammonites from Texas and northern Mexico: Texas University, Bulletin, 2748, 143-31.

Buitrón, B.E., 1970. Equinoides del Cretácico Inferior de la región de San Juan Raya-Zapotitlán, Estado de Puebla: Universidad Nacional Autónoma México, Instituto Geología, Paleontología Mexicana, $30,45 \mathrm{p}$.

Buitrón, B.E., 1971, Equinoides del Cretácico del norte de México: Universidad Nacional Autónoma México, Instituto Geología, Paleontología Mexicana, 31, 46 p.

Buitrón, B.E., 1973, Tetragramma gloriae n. sp., equinoide de la Formación San Lucas (Hauteriviano-Aptiano) de la región de Huetamo, Michoacán: Universidad Nacional Autónoma México, Instituto Geología, Paleontología Mexicana, 36, 33-41.

Burckhardt, C., 1906, La faune jurassique de Mazapil avec un appendice sur les fossiles du Crétacique Inférieur: Instituto Geológico de México, Boletín, 23, 211 p.

Burckhardt, C., 1912, Faunes jurassiques et crétaciques de San Pedro del Gallo: Instituto Geológico de México, Boletín, 29, 264 p.

Burckhardt, C., 1919, Faunas jurásicas de Symon (Zacatecas) y faunas cretácicas de Zumpango del Río, Guerrero. Tomo I. Texto: Instituto Geológico de México, Boletín, 33, 135 p.

Burckhardt, C., 1925, Faunas del Aptiano de Nazas (Durango): Instituto Geológico de México, Boletín, 45, 71 p.

Burckhardt, C., 1927, Cefalópodos del Jurásico Medio de Oaxaca y Guerrero: Instituto Geológico de México, Boletín, 47, 106 p.

Cantú-Chapa, A., 1967, El límite Jurásico-Cretácico en Mazatepec, Puebla (México): Instituto Mexicano del Petróleo, Sección Geología, Monografía 1, Publicación 7AI-II, 3-24

Cantú-Chapa, A., 1968, Sobre una asociación Proniceras-Durangites "Hildoglochiceras" del noroeste de México: Revista del Instituto Mexicano del Petróleo, Sección Geología, Monografía N.2, 1926.

Carranza-Castañeda, O., 1978, Nuevas investigaciones sobre la Fauna Rancho El Ocote Plioceno medio de Guanajuato, México. Informe Preliminar: Universidad Nacional Autónoma de México, Instituto de Geología, Revista, 2(2), 163-166.

Carreño, A. L., Montellano-Ballesteros, M., (eds.), 1997, Extinción masiva del límite Cretácico-Terciario; mitos y realidades: Unión Geofísica Mexicana, Monografía 4, 174 p.

Castillo, A. del, 1869, Clasificación y datos sobre los mamíferos fósiles encontrados en el Valle de México: Deutsche Geologische Gessellschaft, Zeitschrift, 21, 479-482.

Castillo, A. del, 1879, Adelantos de la paleontología y geología del Valle de México: El Minero Mexicano, 484-485.

Castillo, A. del, Aguilera, J. G., 1895, Fauna fósil de la Sierra de Catorce, San Luis Potosí: Comisión Geológica de México (Instituto Geológico de México), Boletín, 1, 55 p.

Cole, W. S., 1927, A foraminiferal fauna from the Guayabal Formation in Mexico: American Paleontology, Bulletin, 14(51), 1-46.

Cole, W. S., 1928a, A foraminiferal fauna from the Chapapote Formation in Mexico: American Paleontology, Bulletin, 14(53), 199-219.

Cole, W. S., 1928b, A new Lepidocyclina from the upper Oligocene of Mexico: American Paleontology, Bulletin, 14(53), 221-222.

Cole, W. S., Guillespie, R., 1930, Some small foraminifera from the Mesón Formation of Mexico: American Paleontology, Bulletin, 15(57b), 123-142.

Comission Scientifique du Mexique, 1867, Archives de la Commision Scientifique du Mexique, 3 vols. Paris, Imprimérie Impériale.

Conrad, T. A., 1857, Description of Cretaceous and Tertiary fossils, in 
Emory, W.H., Report of the United States and Mexican Boundary Survey, made under the Direction of the Secretary of the Interior: Washington, D.C., U.S. Department of Interior, 1(2), 141-174.

Cope, E. D., 1872, On two extinct forms of Physistomi of the Neotropical region: Proceedings of the American Philosophical Society, 12, 52-55.

Cope, E. D., 1884, The extinct Mammalia of the Valley of Mexico: Proceedings of the American Philosophical Society, 22(117), 1-21.

Cope, E. D., 1886a, Report of the coal deposits near Zacualtipan in the State of Hidalgo, Mexico [and description of mammalian fossils from Tehuichila, Veracruz]: Proceedings of the American Philosophical Society, 23, 146-151.

Cope, E. D., 1886b, On two new species of three-toed horses from the upper Miocene, with remarks on the fauna of the Ticholeptus Beds: Proceedings of the American Philosophical Society, 23, 357-361.

Cope, E. D., 1893, Description of a lower jaw of Tetrabelodon shepardii Leidy: Proceedings of the Academy of Natural Science, Philadelphia, 45, 202-204

Cotteau, M., 1890, Note sur quelques Échinides du terrain crétacé du Mexique: Société Géologique de France, Bulletin Série 3, 18, 292-299.

Cuatáparo, J. N., Ramírez, S., 1875, Descripción de un mamífero fósil de especie desconocida, perteneciente al género "Glyptodon", encontrado entre las capas post-terciarias de Tequixquiac, en el Distrito de Zumpango: Sociedad Mexicana Geografía Estadística, Boletín serie 3(2), 354-362.

Cushman, J. A., 1920, The American species of Orthophragmina and Lepidocyclina: U. S. Geological Survey Professional Paper, 125D, 39-105.

Cushman, J.A., 1925, New Foraminifera from the upper Eocene of Mexico: Contributions Cushman Laboratory Foraminiferal Research, 1(1), 4-9.

Cushman, J. A., 1926, Some Foraminifera from the Méndez Shale of eastern Mexico: Contributions Cushman Laboratory Foraminiferal Research, 2(1), 16-26.

Cushman, J. A., 1927, Some characteristic Mexican fossil foraminifera: Journal Paleontology, 1(2), 147-172.

Dugès, A., 1882, Nota sobre un fósil de Arperos, Estado de Guanajuato: El Minero Mexicano, 9(20), 233-235.

Dugès, A., 1891, Platygonus alemanii: La Naturaleza, 2(1), 16-18.

Dunbar, C. O., 1939, Permian fusulines from Sonora: Geological Society of America, Bulletin, 50, 1745-1760.

Dunbar, C. O., 1944, Pte. 2, Permian and Pennsylvania (?) Fusulines, en King, R. E., y colaboradores (eds.), Geology and Paleontology of the Permian area northwest of Las Delicias, southwestern Coahuila, Mexico: Geological Society of America Special Paper, 52, 35-48.

Erben, H. K., 1954, Dos amonitas nuevos y su importancia para la estratigrafía del Jurásico Inferior de México: Universidad Nacional Autónoma de México, Paleontología Mexicana, 1, 23 p.

Erben, H. K., 1956, El Jurásico Inferior de México y sus amonitas: México, 20 Congreso Geológico Internacional, 393 p.

Espinosa-Arrubarrena, L., Applegate, S. P., 1996, A possible model for the paleoecology of the vertebrate bearing beds in the Tlayúa quarries, near Tepexi de Rodríguez, Puebla, Mexico: en Arratia, G., Viohl, G., (eds.), Mesozoic Fishes-Systematics and Paleoecology: München, Alemania, 539-550.

Felix, J., Lenk, H., 1889-1899, Beiträge zur Geologie und Paläontologie der Republik Mexico: Leipzig, Stuttgart, Verlag von Arthur Felix; und E. Schweizerbart'sche Verlagshandlung (E. Nägele), 3 v.

Ferrusquía-Villafranca, I., 1969, Rancho Gaitán Local Fauna, Early Chadronian, northeastern Chihuahua: Sociedad Geológica Mexicana, Boletín, 30(2), 99-138.

Ferrusquía-Villafranca, I., 1978, Distribution of Cenozoic vertebrate faunas in Middle America and probles of migration between North and South America: Universidad Nacional Autónoma de México, Instituto de Geología, Boletín, 101, 193-329.

Freudenberg, W., 1910, Die Säugetierfauna des Pliocäns und Postpliocäns von Mexiko. I. Carnivoren: Geologische Paläontologische, Abh, 9(3), 195-231.
Freudenberg, W., 1921, Geologie von Mexiko: Verlag von Gebrüder Bomtraeger, Berlín, $232 \mathrm{p}$.

Freudenberg, W., 1922, Die Säugetierfauna des Pliocäns und Postpliocäns von Mexiko. II. Tiel; Mastodonten und Elefanten: Geologische Paläontologische, Abh, 14(3), 103-176.

Gabb, W. M., 1864, On Cretaceous fossils from Sahuaripa Valley, State of Sonora, Mexico, discovered by August Remond: Proceedings of the California Academy of Natural Sciences, 3(2), 153-154.

Gabb, W. M., 1869, Descriptions of Cretaceous fossils collected by A. Remond, at Arivechi, Sonora, Mexico [Notes on some Mexican Cretaceous fossils, with descriptions of new species]: Geological Survey of California, Paleontology of California, 2 (3), 257-276.

Gabb, W. M., 1872, Notice of a collection of Cretaceous fossils from Chihuahua, Mexico: Proceedings of the Academy of Natural Sciences of Philadelphia, 24(2), 263-265,

Galeotti, H., 1839, Notice sur le calcaire crétacé des environs de Jalapa, au Mexique: Bulletin de la Société Géologique de France, Série $1,10,32-39$.

Galloway J. J., Morrey, M., 1931, Late Cretaceous Foraminifera from Tabasco, Mexico: Journal of Paleontology, 5(4), 329-353.

Gío-Argáez, R., 2004, Los fósiles: Ciencia, 55, 4-7.

Grisebach, A., 1883, La vegetación de México, clima, formaciones vegetales y regiones. Centro de vegetación: La Naturaleza, primera serie, 6, 251-279.

Hernández-Rivera, R., 1997, Mexican dinosaurs, en Currie P. J., Padian K., (eds.) Encyclopedia of Dinosaurs: Academic Press, U. S. A., 433-437.

Hertlein, L. G., 1925, Pectens from the Tertiary of Lower California: Proceedings of the California Academy of Sciences, ser 4, 14(1), $35 \mathrm{p}$.

Hertlein, L. G., 1934, Pleistocene mollusks from the Tres Marias Islands, Cedros Island and San Ignacio Lagoon, Mexico: Southern California Academy of Sciences, Bulletin, 33, 59-73.

Herrera y Tordesillas, A., 1615, Historia general de los hechos de los Castellanos en las Islas y Tierra firme del Mar Océano, 9 vols., Madrid: Imprenta Real.

Imlay, R. W., 1937a, Lower Neocomian fossils from the Miquihuana region, Mexico: Journal of Paleontology, 11(7), 552-574.

Imlay, R. W., 1937b, Stratigraphy and paleontology of the Upper Cretaceous beds along the eastern side of the Laguna de Mayrán, Coahuila, Mexico: Geological Society of America, Bulletin, 48(12), $1785-1872$.

Imlay, R. W., 1938, Ammonites of the Taraises Formation of northern Mexico: Geological Society of America, Bulletin, 49(4), 539602 .

Imlay, R.W., 1939, Upper Jurassic ammonites from Mexico: Geological Society of America, Bulletin, 50(1), 1-77.

Imlay, R.W., 1940, Neocomian faunas of northern Mexico: Geological Society of America, Bulletin, 51(1), 117-190.

Maldonado-Koerdell, M., 1948, Nuevos datos geológicos y paleontológicos sobre el Triásico de Zacatecas (México): Escuela Nacional Ciencias Biológicas, Anales, 5(3-4), 291-306.

Maldonado-Koerdell, M., 1950, Faunas del alto Cretácico Superior, del Paleoceno y del Eoceno inferior y medio de Chiapas, México: Escuela Nacional de Ciencias Biológicas, Anales, 6(1-4), 181220.

Maldonado-Koerdell, M., 1953a, Status of micropaleontology in Tabasco and Chiapas (Mexico): Contributions from the Cushman Foundation for Foraminiferal Research, 4(4), 109-116.

Maldonado-Koerdell, M., 1953b, Los equinoides regulares del Mesozoico de México: Anales Escuela Nacional Ciencias Biológicas, 7(1-4), 15-44.

Martínez-Hernández, E., Ludlow-Wiechers, B., Sánchez,-López, M., 1980, Catálogo palinológico de la Cuenca Fuentes-Río Escondido, v. 1. Esporas monoletes, esporas triletes, granos de polen sulcados: Serie Técnica Comisión Federal de Electricidad, 6, 241 p.

Martins, C., 1871, Las poblaciones vegetales, su origen, su composición y sus emigraciones: La Naturaleza, primera serie, 2, 18-27; 148$154 ; 241-250$.

Meyer, H., von, 1840a, Neue Gattungen Fossiler Krebse aus Gebilden vom 
Bunten Sandstein bis in die Kreide. Stuttgart: Schweizerbart.

Meyer, H., von, 1840b, Über die Sammlung von Mexikanischen Antiquitäten, Mineralien und Petrefakten: Neues Jahrbuch für Mineralogie, Geologie und Paläontologie 1840, 576-587.

Montellano-Ballesteros, M., 1999, Mexico and Central America en, Singer R. (ed.), Encyclopedia of Paleontology, Fitzroy Dearborn Publishers, Chicago, EUA, 2 M-Z, 728-734.

Mooser, O., 1958, La Fauna "Cedazo" del Pleistoceno en Aguascalientes: Universidad Nacional Autónoma de México, Instituto de Biología, Anales, 29, 409-452.

Mooser, O., 1959, Un équido fósil del género Neohipparion de la Mesa Central de México: Universidad Nacional Autónoma de México, Instituto de Biología, Anales, 30, 375-388.

Mooser, O., 1963, Neohipparion monias n. sp. équido fósil del Plioceno de la Mesa Central de México: Universidad Nacional Autónoma de México, Instituto de Biología, Anales, 34(1-2), 393-396.

Morán, D., 1999, Presentation of the 1999 Harroll L. Strimple Award of the Aranguti Family: Journal of Paleontology, 74(4), 761-762.

Müllerried, F. K. W., 1931, Chiapasella, un paquiodonto extrañísimo de la América: Universidad Nacional Autónoma de México, Instituto de Biología, Anales, 2(3), 243-254.

Müllerried, F. K. W., 1933, Estudios paleontológicos y estratigráficos en la región de Tehuacán, Puebla: Universidad Nacional Autónoma de México, Instituto de Biología, Anales, 4(2), 79-93.

Müllerried, F. K. W., 1944, Geología, estratigrafía y paleontología de la región de Cacahuamilpa (México): Escuela Nacional de Ciencias Biológicas, Anales, 3(3-4), 463-484.

Müllerried, F. K. W., 1945, El edestido Helicoprion, encontrado por primera vez en México en el Estado de Coahuila: Ciencia, 5(5-6), 208-212.

Müllerried, F. K. W., 1947, Paleobiología de la caliza de Córdoba y Orizaba, Ver. (México): Universidad Nacional Autónoma de México, Instituto de Biología, Anales, 18(2), 361-462.

Müllerried, F. K. W., 1951, Algunos fósiles marinos del Terciario inferior y medio de Palenque, Chiapas: Sociedad Mexicana Historia Natural, Revista, 12(1-4), 209-227.

Nathorst, A., 1899, Pflanzenresten aus dem Neocom von Tlaxiaco, en Félix, J., Lenk, H., Beiträgezur Geologie und Paläontologie der Republik Mexico, Theil 2: Leipzig, Stuttgart, Verlag von Arthur Felix; und E. Schweizerbart'sche Verlagshandlung (E. Nägele), 51-54.

Noé, A. C., 1937, Migración y evolución de las faunas y flores fósiles americanas y sus relaciones estratigráficas aparentes en las costas del Atlántico y Pacífico: Sociedad Geológica Mexicana, Boletín 10(1-2), 61-73.

Nuttall, W. L. F., 1928, Notes on the Tertiary Foraminifera of southern Mexico: Journal of Paleontology, 2(4), 372-376.

Nuttall, W. L. F., 1930, Eocene Foraminifera from Mexico: Journal of Paleontology, 4(3), 271-293.

Nuttall, W. L. F., 1932, Lower Oligocene Foraminifera from Mexico: Journal of Paleontology, 6(1), 3-35.

Nyest, H., Galeotti, H., 1840, Description de quelques fossiles du calcaire jurassique de Tehuacán, au Mexique: de Académie Royale des Sciences et Belles-Lettres de Bruxeles, Bulletins, 7(2), 212-221.

Obregón de la Parra, J., 1958, Parvicarinina mexicana, una nueva especie de foraminífero del Paleoceno de Nuevo León, México: Asociación Mexicana de Geólogos Petroleros, Boletín, 10(7-8), 643-648.

Obregón de la Parra, J., 1959, Foraminíferos de la Formación La Peña: Asociación Mexicana de Geólogos Petroleros, Boletín, 11 (3-4), 135-153.

Obregoso, J., de 1931, Reconocimiento del Istmo de Tehuantepec, hecho el año de 1825 por el Ciudadano Juan de Obregoso, Jalapa: Impreso por Blanco y Aburto en la Oficina del Gobierno.

Ochoterena, H., 1960, Variación intraespecífica en Parathyridina mexicana n. sp., terebratúlido del Oxfordiano de México: Universidad Nacional Autónoma de México, Instituto de Geología, Paleontología Mexicana, 9, $40 \mathrm{p}$.

Ochoterena, H., 1963, Amonitas del Jurásico Medio y del Calloviano de México, I. Parastrenoceras gen. nov.: Universidad Nacional Autónoma de México, Instituto de Geología, Paleontología
Mexicana, 16, $26 \mathrm{p}$.

Ochoterena, H., 1966, Amonitas del Jurásico Medio de México, II. Infrapatoceras gen. nov.: Universidad Nacional Autónoma de México, Instituto de Geología, Paleontología Mexicana, 23, 18 p.

Owen, F. R. S., 1869, On remains of a large extinct Lama (Palauchenia magna, Ow.) from Quaternary deposits in the Valley of Mexico: Proceedings Royal Society of London, 160, 65-77.

Perrilliat, M. C., 1972, Monografía de los moluscos del Mioceno medio de Santa Rosa, Veracruz, México. Parte I. (Gasterópodos: Fissurellidae a Olividae): Universidad Nacional Autónoma de México, Instituto de Geología, Paleontología Mexicana, 32, 130 p.

Perrilliat, M. C., 1973, Monografía de los moluscos del Mioceno medio de Santa Rosa, Veracruz, México. Parte II. (Gasterópodos: Mitridae a Terebridae) Universidad Nacional Autónoma de México, Instituto de Geología, Paleontología Mexicana, 35, 96 p.

Perrilliat, M. C., 1974a, Monografía de los moluscos del Mioceno medio de Santa Rosa, Veracruz, México. Parte III. (Gasterópodos: Pyramidellidae a Siphonariidae): Universidad Nacional Autónoma de México, Instituto de Geología, Paleontología Mexicana, 37, 1- 46.

Perrilliat, M. C., 1974b, Monografía de los moluscos del Mioceno medio de Santa Rosa, Veracruz, México. Parte IV. (Escafópodos: Dentaliidae, Siphonodentaliidae): Universidad Nacional Autónoma de México, Instituto de Geología, Paleontología Mexicana, 37, 51-64.

Perrilliat, M. C., 1976, Monografía de los moluscos del Mioceno medio de Santa Rosa, Veracruz, México. Parte V. (Pelecípodos: Nuculidae a Limidae): Universidad Nacional Autónoma de México, Instituto de Geología, Paleontología Mexicana, 42, 53 p.

Perrilliat, M. C., 1977, Monografía de los moluscos del Mioceno medio de Santa Rosa, Veracruz, México. Parte VI. (Pelecípodos: Lucinidae a Solecurtidae): Universidad Nacional Autónoma de México, Instituto de Geología, Paleontología Mexicana, 43, 60 p.

Perrilliat, M. C., 1984, Monografía de los moluscos del Mioceno medio de Santa Rosa, Veracruz, México, Parte VII, (Pelecípodos: Dreissenidae a Verticordiidae): Universidad Nacional Autónoma de México, Instituto de Geología, Paleontología Mexicana, 48, 88 p.

Polaco, O. J., Arroyo-Cabrales, J., 1991, Colección paleontológica de la Subdirección de Servicios Académicos: Instituto Nacional de Antropología e Historia, Memoria de la II Reunión Nacional de Colecciones Paleontológicas, México, D. F., 55-57.

Reyeros de Castillo, M. M., 1974, Corales del Jurásico Superior de Chihuahua: Universidad Nacional Autónoma de México, Instituto de Geología, Paleontología Mexicana, 40, 1-21.

Reyeros de Castillo, M. M., 1978, Invertebrados (Porífera y Coelenterata) del Jurásico Tardío del Estado de San Luis Potosí: Universidad Nacional Autónoma de México, Instituto de Geología, Revista, 2(1), 69-74.

Río, A. M., del, 1795, Elementos de Orictognosia; o del Conocimiento de los fósiles dispuestos según los principios de A. G. Werner, para el uso del Real Seminario de Minería de México, 2 vols: Mexico City, Zúñiga y Ontiveros.

Sahagún, Bernardino de, Fray, (1558-1569), Historia General de las Cosas de la Nueva España: Madrid, Ed. F. Del Paso y Troncoso, 4 vols.

Salas, E., Palacios-Fest, M. R., 1982, Nuevas esporas estriadas del Mesozoico del sureste de México: Instituto Mexicano del Petróleo, Revista, 14(4), 88-98.

Salmerón, U. P., 1972, Mutación entre los géneros Pararotalia y Miogypsinoides: Instituto Mexicano del Petróleo, Revista, 4(3), 5-27.

Sansores, J. C., Flores-Covarrubias, C., 1972, Descripción de seis nuevas especies de foraminíferos bentónicos de la Cuenca Salina del Istmo de Tehuantepec: Instituto Mexicano del Petróleo, Revista, 4(4), 77-83.

Silva-Pineda, A., 1970, Plantas fósiles del Jurásico medio de la región de Tezoatlán, Oaxaca: Sociedad Geológica Mexicana, Libreto guía de la excursión México-Oaxaca, 129-153.

Silva-Pineda, A., 1978, Contribuciones a la paleobotánica del Jurásico de México, Pte. 1: Universidad Nacional Autónoma de México, Instituto de Geología, Paleontología Mexicana, 44, 16 p. 
Torrubia, J., 1754, Apparato para la historia natural Espagnola: Contiene muchas dissertaciones physicas especialmente sobre el Diluvio. v. 1, Madrid: Jordejuela.

Trejo, M., 1969, Conusphaera mexicana, un nuevo cocolitofórido del Jurásico Superior de México: Instituto Mexicano del Petróleo, Revista, 1(4), 5-15.

Trejo, M., 1972, Nuevos tintínidos del Aptiano superior de México: Instituto Mexicano del Petróleo, Revista, 4(3), 80-87.

Vaughan, T. W., Cole, W. S., 1936, New Tertiary Foraminifera of the genera Operculina and Operculinoides from North America and the west Indies: Proceedings U. S. Natural Museum, 83(2996), 487-496.

Weber, R., 1972, La vegetación maestrichtiana de la Formación Olmos de Coahuila, México: Asociación Mexicana de Geólogos Petroleros, Boletín, 33, 5-19.

Weber, R., 1973, Salvinia coahuilensis sp. nov. del Cretácico superior de México: Ameghiniana, 10(2), 173-190.
Weber, R., 1975, Aachenia knoblochi n. sp. an interesting conifer from the Upper Cretaceous Olmos Formation of northeastern Mexico: Palaeontolographica. Abteilung B: Palaeophytologie Verlagsbuch Handlung, 152, 76-83.

Weyland, H., 1937, Beiträge zur Kenntnis der Rheinischen Tertiärflora: Paleontographica Abt. B. 83, 67-122.

White, M. P., 1928a, Some index Foraminifera of the Tampico embayment area of Mexico, Pt. 1: Journal of Paleontology, 2(3), 177-215.

White, M. P., 1928b, Some index Foraminifera of the Tampico embayment area of Mexico, Pt. 2: Journal of Paleontology, 2(4), 280-317.

Manuscrito recibido: Agosto 20, 2004

Munsucrito corregido recibido: Abril 20, 2005

Manuscrito aceptado: Mayo 11, 2005 